\title{
Japanese lunar robotics exploration by co-operation with lander and rover
}

\author{
Takashi Kubota ${ }^{1, *}$, Yasuharu Kunir ${ }^{2}$, Yoji Kuroda ${ }^{3}$ and Working Group \\ ${ }^{1} I S A S / J A X A$, 3-1-1, Yoshinodai, Sagamihara, Kanagawa 229-8510, Japan. \\ ${ }^{2}$ Chuo University, 1-13-27, Kasuga, Bunkyo-ku, Tokyo 113-8551, Japan. \\ ${ }^{3}$ Meiji University, 1-1-1, Higashi-mita, Tama-ku, Kawasaki 214-8571, Japan. \\ *e-mail:kubota@nnl.isas.jaxa.jp
}

Unmanned mobile robots for surface exploration of the Moon or planets have been extensively studied and developed. A lunar rover is expected to travel safely in a wide area and explore in detail. Japanese lunar robotics exploration is under study to conduct an unmanned geological survey in the vicinity of central peaks of impact craters for investigation of the sub-surface materials. This will give us the key information to study the lunar inner structure and understand the Moon's origin and evolution as well as to investigate the evolution of magma ocean and later igneous processes. To carry out the geological exploration in the central peak, lander and rover co-operative exploration is proposed. The working group has been conducting feasibility study of advance technologies. This paper addresses an overview of lunar exploration with lander and rover and also enumerates future technologies to be established.

The rover R\&D group has developed an innovative science micro rover with a new mobility system and a lightweight manipulator. The design and implementation of a science rover for the near future lunar missions requiring long traverses and scientific observations are described and some experimental results are presented.

\section{Introduction}

In Japan, a new lunar exploration mission including lander and rover is under study. The mission will follow up SELENE (Selenoligical Engineering Explorer), a lunar global remote sensing mission scheduled for 2006. The main mission for lunar robotics exploration in post SELENE mission (Nakatani et al 2003) is to demonstrate the technologies for lunar or planetary surface exploration. They will cover pin-point landing technology, reliable landing scheme with obstacle avoidance (Nishiguchi 2002), safe landing mechanism on rough terrain, exploration rover and tele-science technology. The following science experiments will also be conducted in the robotics mission (Sasaki et al 2004a). Geologic survey will be conducted in the vicinity of central peaks of impact craters to investigate the exposed sub-surface materials. In situ analyses of the surface rocks and soils will also be performed with a special emphasis on the investigation of their structure and composition by cutting or grinding the collected samples. The characterization of the site can be done by multi-band imaging and X-ray and gamma-ray spectrometry. This will provide key information in understanding the lunar inner structure and the origin and evolution of the moon, as well as the evolution of magma ocean and subsequent igneous processes.

For geological exploration of the central peak, the lander and rover co-operative exploration is under study as shown in figure 1. The working group has been conducting the feasibility study of advanced technologies for lunar robotics exploration. Unmanned mobile robots are required for

Keywords. Lunar robotics exploration; central peak exploration; lander and rover co-operation; micro rover; micro manipulator. 


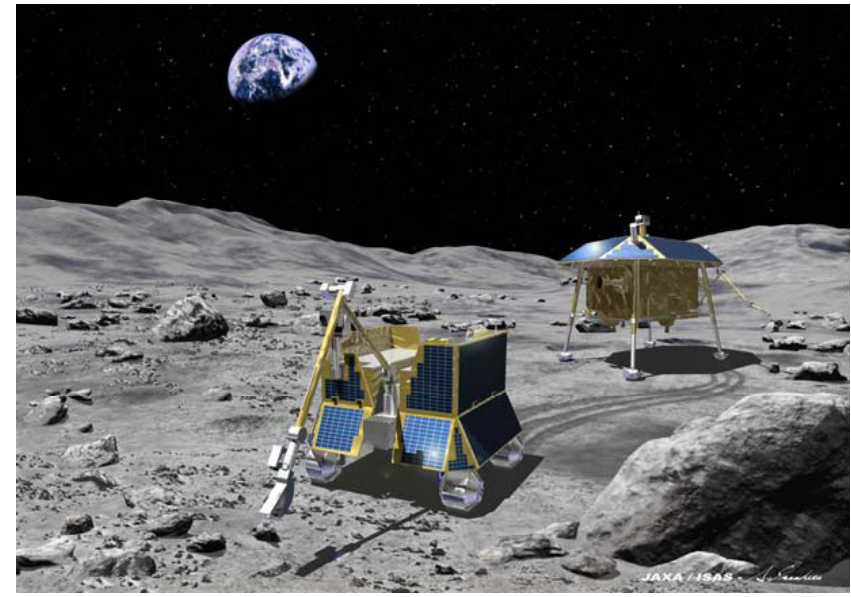

Figure 1. Lander-rover exploration.

detailed surface exploration of the moon, because rovers would be able to travel safely over a long distance and make observations with the aid of some scientific instruments. Therefore the rover R\&D group has developed an innovative science micro rover with a new mobility system and a lightweight manipulator. This paper describes the design and implementation of a science rover for lunar missions requiring long traverses and rover-based scientific observation.

\section{Lander-rover exploration}

\subsection{Central peak exploration}

To understand the formation of lunar crust through early differentiation (Nozette 1996), it is important to know the current subsurface layered structure of the lunar crust. Apollo mission brought back various rock fragments, some of which should have come from the lunar interior. Although they are different from the surface materials, their location of formation within the Moon is not known. In the central peaks or peak rings of large impact craters, underlying crustal and possibly upper mantle materials should have been uplifted from beneath the surface (Wieczorek and Phillips 2000). Thus, central peaks are 'windows' to the lunar interior. Using Clementine UV-VIS multispectral data, Tompkins and Pieters (1999) analyzed central peaks of 109 impact craters all over the moon. Some of them show olivine-rich signature, which may represent the upper mantle materials. Those include the craters Copernicus, Crookes, Keeler, Langrenus, Theophilus, and Tsiolkovski. Most of them are located at low latitudes (within $\pm 20^{\circ}$ ). Among them, Copernicus, Langrenus, and Theophilus are easy to visit because they are on the nearside of the Moon. Since they have exposed olivine-rich mantle material, material from the lower crust also must be explored there. Therefore, if some area of the central peak region within one of these craters is investigated directly, a precise layering model of the lunar crust - upper mantle can be constructed.

\subsection{Mission scenario}

A possible scenario of investigation of the crater interior or central peak can be as follows (Sasaki et al 2004b).

- Landing on the lava plain within about $500 \mathrm{~m}$ of the flank of the central peak.

- Remote observation of the central peak by a multi-spectral camera on the lander.

- Observation of the area surrounding the lander.

- Deploying the rover and performing short-term observations around the lander.

- Mid-distance (about $100 \mathrm{~m}$ ) exploration by the rover with multi-band observation of rocks and soils and gamma-ray observation of the surface.

- Approaching the central peak and conducting scientific measurements (multi-spectral observation of rocks and soils, gamma-ray observation of the surface) on the way.

- At the foot of the central peak, observing and analyzing rocks from the central peak.

- Climb-up (short distance) the flank of the central peak.

- Come-back to the lander. Samples are put into a mini-lab in the lander for analysis.

\subsection{Lander-rover co-operation}

To achieve efficient and effective geological exploration, the lander and rover co-operation is proposed. Scientific instruments should be installed in both the lander and the rover. The rover performs basic characterization of the surface rock and regolith and the detailed observation of samples is done on the lander after the rover returns to the lander. The rover should travel in wide areas and carry back to the lander at least five samples of $2-3 \mathrm{~cm}$. If there is some possibility to land on top of the central peak, the lander may directly access the central peak materials. Even in this case, the deployment of the rover is preferable because investigations can be extended to larger areas where even small surface geological diversity can be observed.

For the lander-rover co-operative geologic mission, some science instruments have been considered. The rover should have macro/micro camera with spectroscopy and gamma-ray spectrometer in addition to navigation cameras. Not only determination of elemental and mineral compositions but 


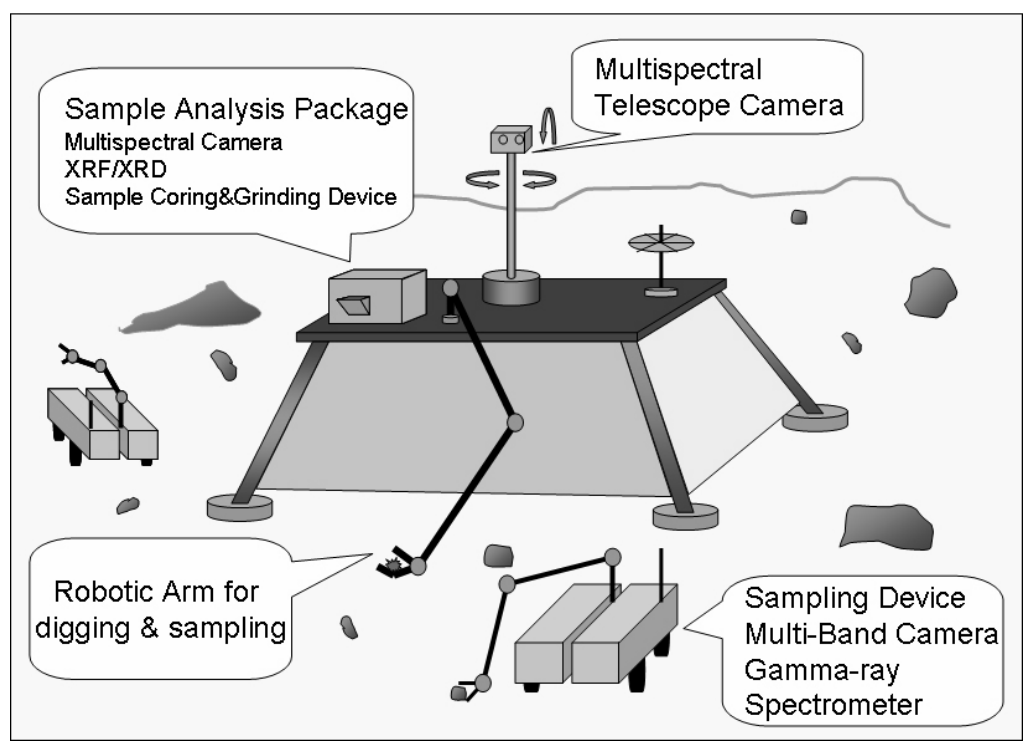

Figure 2. Lander-rover co-operation.

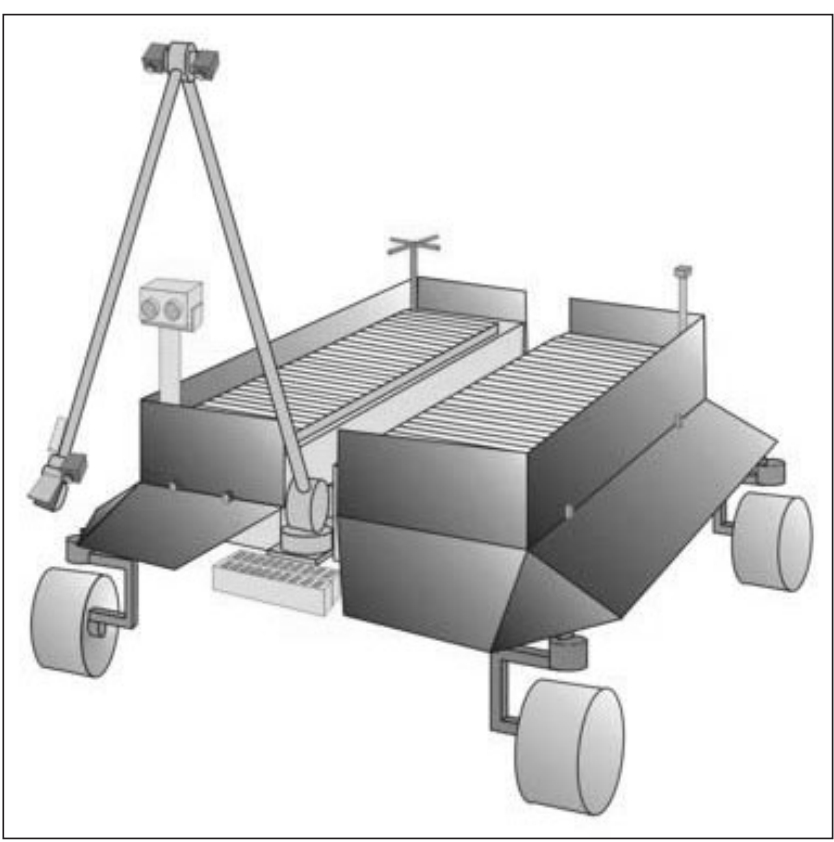

Figure 3. Illustration of designed rover.

also analyses of the mineral textures are desirable, since many of the lunar rocks are impact-induced polymict breccias. Observation of rock fragments in brecciated rocks is necessary. For this purpose, it is desirable that the rover should have a cutting or surface-grinding and coring mechanism for rocks. If extra weight can be accommodated, the rover may also have magnetic instruments to measure the change of surface magnetic properties. Mature soils should have more phases of iron particles, which have strong magnetic effects (Hapke et al 1975).
Table 1. Specifications of designed rover.

\begin{tabular}{ll}
\hline Size & $\left\{\begin{array}{l}0.85 \mathrm{~m} \text { (length) } \\
0.85 \mathrm{~m} \text { (width) } \\
0.60 \mathrm{~m} \text { (height) } \\
\text { About } 30 \mathrm{~kg}\end{array}\right.$ \\
Weight & $\left\{\begin{array}{l}\text { PEGASUS } \\
\text { Wheel diameter } 0.15 \mathrm{~m}\end{array}\right.$ \\
Mobility system & $\left\{\begin{array}{l}\text { Velocity: } 3 \mathrm{~cm} / \mathrm{s} \\
\text { Climbable step: } 0.25 \mathrm{~m} \\
\text { Climbable slope: } 30 \mathrm{deg}\end{array}\right.$ \\
Mobility performance & $\left\{\begin{array}{l}\text { Solar panel: max } 40 \mathrm{~W} \\
\text { Battery: NiCd, Lithium }\end{array}\right.$ \\
Power supply & $\left\{\begin{array}{l}\text { Actuator: max } 6 \mathrm{~W} \\
\text { Computer: max } 7 \mathrm{~W}\end{array}\right.$ \\
Power consumption & $\left\{\begin{array}{l}4 \text { stereo cameras } \\
\text { Manipulator, clinometers, IMU } \\
\text { Science instruments }\end{array}\right.$ \\
Payload &
\end{tabular}

The lander should have a narrow-angle multispectral camera for observation of the surrounding area and probable rover targets. Geological characterization of the central peak from the lander should complement previously obtained remote sensing data. AOTF (Acoustic-Optic Tunable Filter) system has been developed for imaging spectroscopy. Advantages of AOTF over a filter wheel or grating system are high resolution, high speed, random or sequential wavelengths access, no moving parts, compact size, and imaging capabilities. Moreover, since the rover has a sampling mechanism, the lander should have a mini-laboratory (sample analysis package) with an $\mathrm{X}$-ray spectrometer, a microscopic spectroscopy and imaging cameras, possibly using AOTF. 

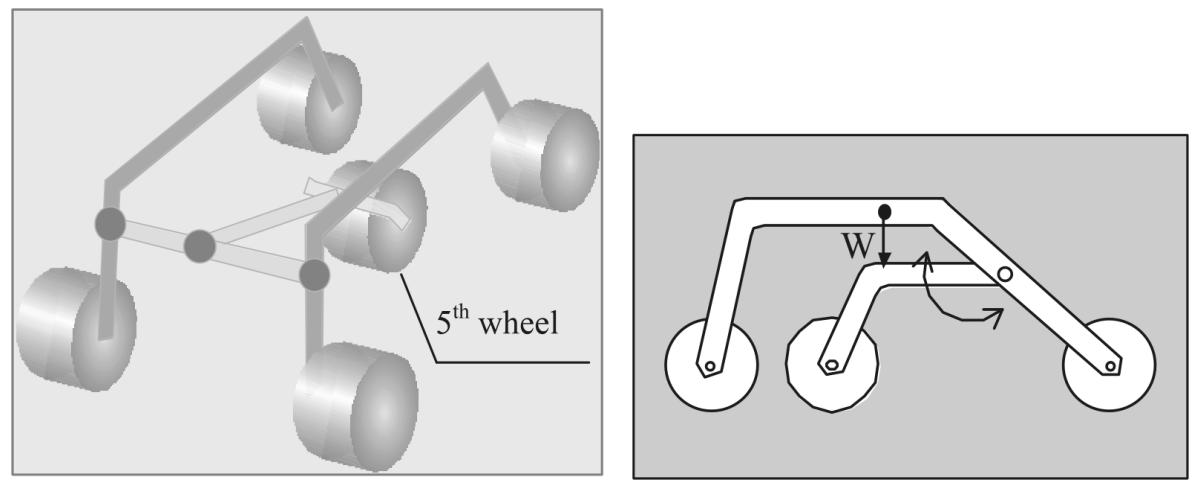

Figure 4. Mechanism of PEGASUS system.

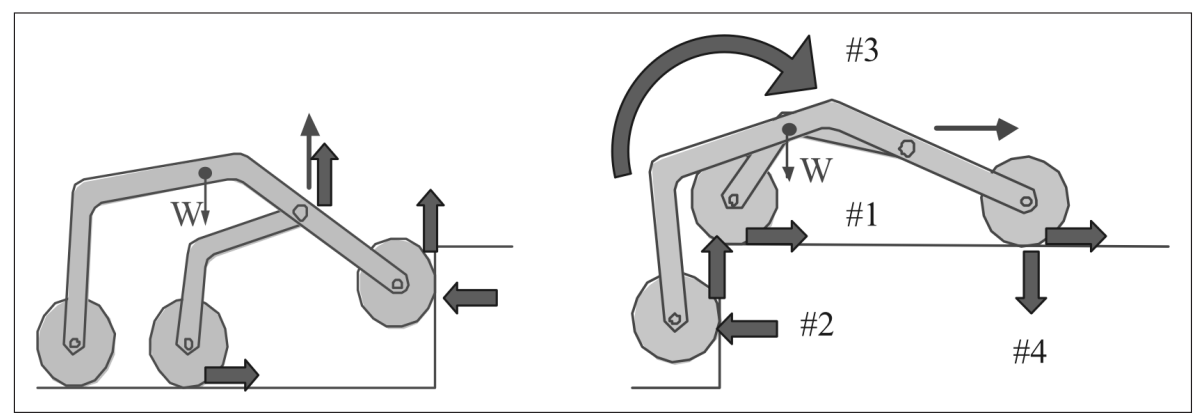

Figure 5. Kinematics of PEGASUS to climb a step.

\section{Rover system}

\subsection{Lunar rover design}

The authors have designed a small rover for lunar robotic exploration mission requiring long traverses and rover-based science experiments. The illustration and the design specification of the developed micro rover are shown in figure 3 and table 1 respectively. The weight of the designed rover is about $30 \mathrm{~kg}$. The rover measures about $0.85 \mathrm{~m}$ wide, $0.85 \mathrm{~m}$ long and $0.60 \mathrm{~m}$ high. The wheel diameter is $0.15 \mathrm{~m}$.

The designed rover is driven by five wheels controlled independently. The steering is controlled by differential of left and right wheels. These wheels are actuated by small DC motors. The velocity of the rover is about $3 \mathrm{~cm} / \mathrm{s}$. The designed rover has the new suspension system called PEGASUS (PEntad Grade Assist SUSpension) (Kubota et al 2001). PEGASUS has high mobility as well as rocker-bogie suspension, with very simple mechanism called only-one-joint architecture. PEGASUS makes it possible for the rover to climb up on the step-like terrain with low energy consumption. The climbable step of the rover is about $0.25 \mathrm{~m}$ and the climbable slope is about $30^{\circ}$. Power is supplied by a solar panel on top of the rover. The rover is also driven by onboard batteries.
Stereo cameras are used for a forward terrain sensor. This rover also has some other CMOS cameras around the body for navigation and scientific observation. The rover is equipped with pitch and roll clinometers for attitude detection and encoders for dead-reckoning. Onboard computers perform sensor data processing and control. The RISCCPUs are dedicated to the function of environment recognition, path planning and navigation.

The designed rover has a communication system to communicate with the ground system. The rover can send images, house-keeping data, and scientific data to the ground system. Operators can control the robot based on image data by teleoperation techniques. The micro rover has a sampling system. The lightweight manipulator with a CMOS camera has been developed, which is attached to the front of the rover. Some of these scientific instruments are under development.

\subsection{PEGASUS system}

Various kinds of the mobility systems for traverse on rough terrain have been proposed. The suspension system is the key issue for realizing high degrees of mobility. NASA/JPL developed rocker-bogie suspension in a series of the project called 'Rocky' (Weisbin 1997). This system consists of a pair of two links called the rocker and the 

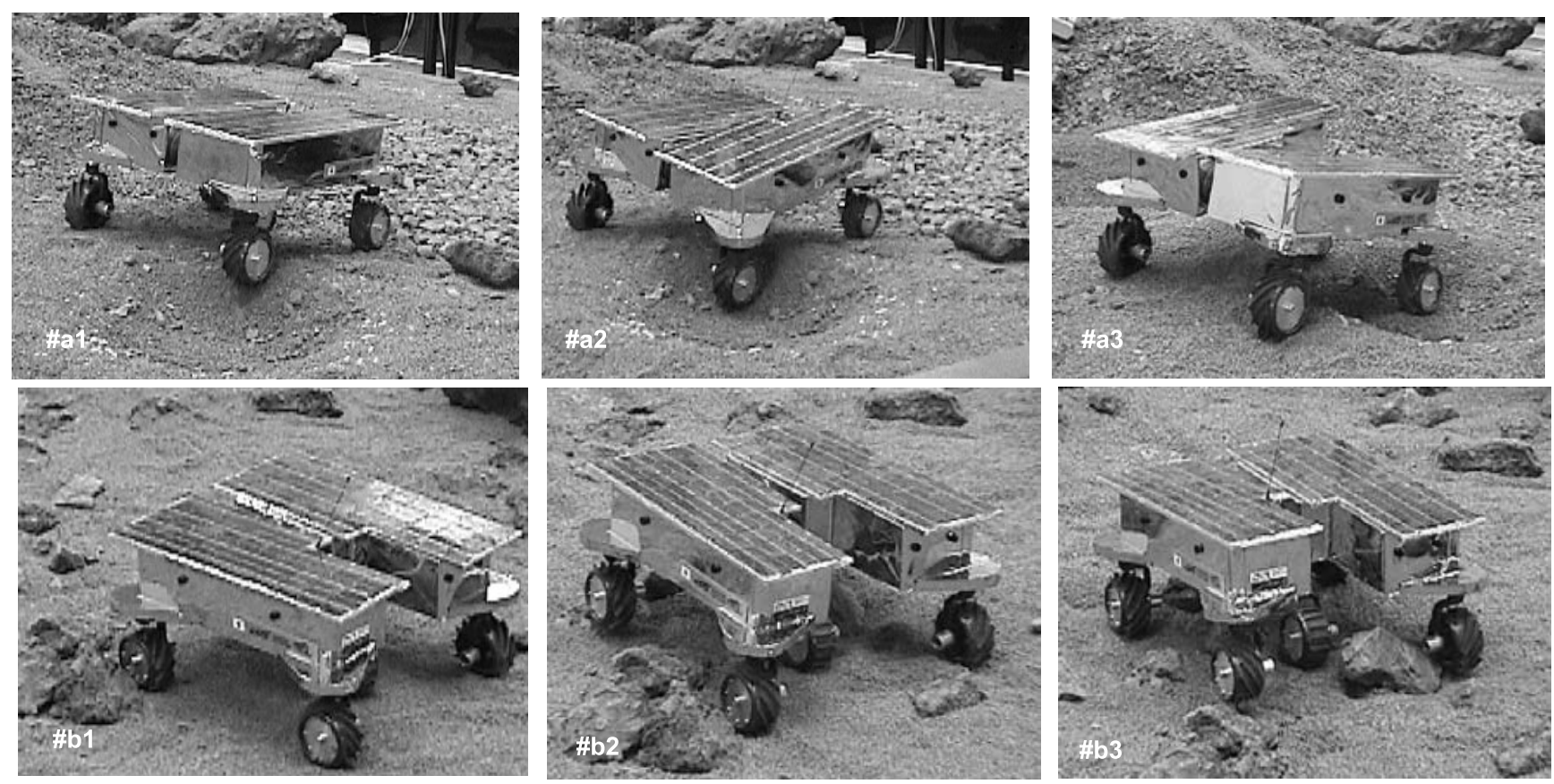

Figure 6. Experimental results on Micro5 mobility.

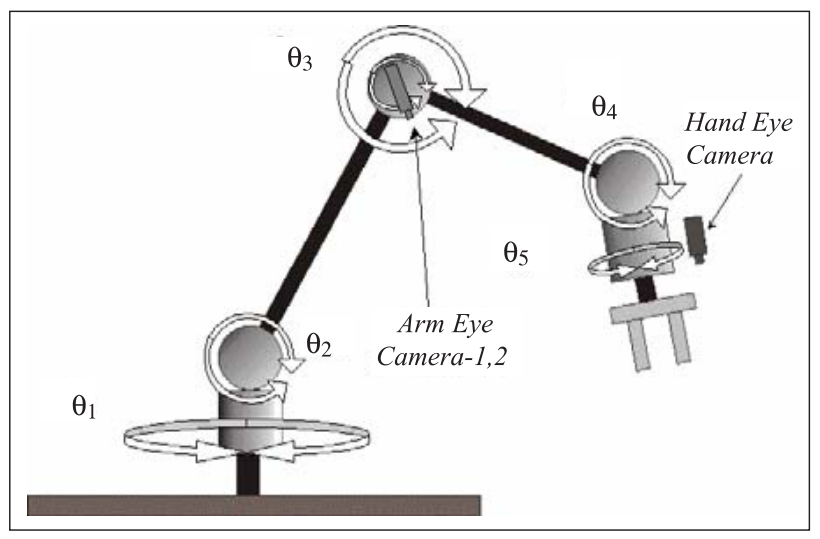

Figure 7. Structure of micro manipulator.

bogie, which are attached to each other by a passive rotary joint. This combination of the rocker and the bogie makes it possible for the rover to smoothly climb rocks 1.5 times its wheel diameter in height. The rocker-bogie suspension system provides extremely high degree of mobility for the rover. However this is not a perfect system for a smaller rover. The rocker-bogie system of Rocky 7 has six wheels. A many-wheels system needs a large number of motors and gears, which increases the weight. Another problem comes from the structure that wheels are attached at the end of the long links and the links are connected by rotary joints as a chain. As a consequence, strong stress would act on the links and the joints, even if a small force is acted on the wheels and the structure has to be made heavier to endure the strong stress.
A small long-range rover is required to have both a simple and lightweight mechanism like four-wheel drive system and a high degree of mobility like rocker-bogie suspension system. In order to achieve these contradictory requirements, we have developed a suspension system as shown in figure 4 . The proposed suspension system PEGASUS consists of a conventional four-wheel drive system and a fifth active wheel connected by a link. The fifth wheel, which is attached to the end of the link, is attached to the body with a passive rotary joint. This joint has no restriction from spring or actuator and can move freely. The proposed system is designed to distribute the load of weight equally to all the five wheels whenever the rover climbs up or down. This implies that the fifth wheel supports the load on the front wheels when the front wheels climb up rocks, and it also supports the load on the rear wheels when the rear wheels climb up the rocks.

When the rear wheel climbs a step, as shown in figure 5 , forward force generated by the traction of the fifth wheel acts backward as \#2. These forces produce nose-dive moment \#3, then the moment turns to a vertical force of the front wheel \#4 to support traction. By this mechanism, when the rover moves forward (corresponding to right direction in figure 5), this mechanism works at maximum performance. When the rover moves backward, the mechanism works as a conventional four-wheel drive system. Most of the times during the mission, the rover moves forward. Therefore, the unidirectional characteristic is not a 

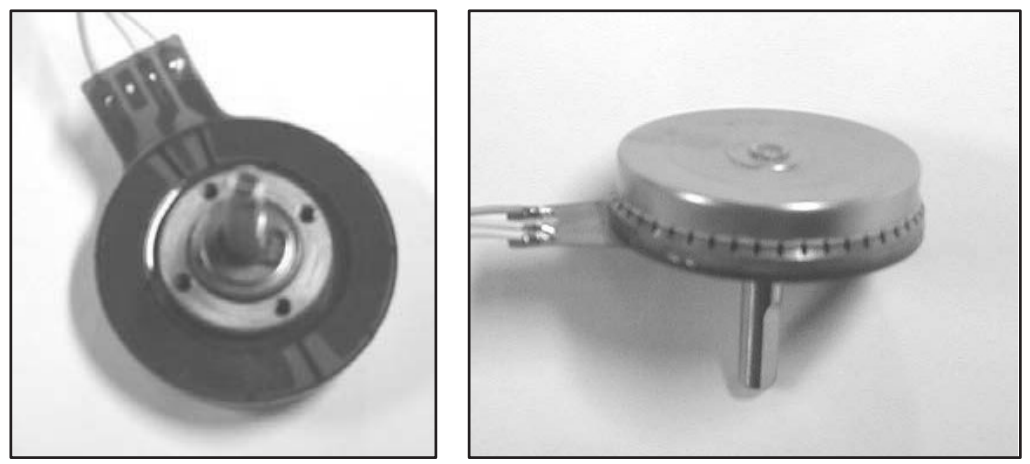

Figure 8. Ultra-Sonic Motor (USM).

Table 2. USM Specifications.

\begin{tabular}{ll}
\hline Rated speed & $250 \mathrm{rpm}$ \\
Rated torque & $0.5 \mathrm{kgf}-\mathrm{cm}$ \\
Rated power & $1.3 \mathrm{~W}$ \\
Maximum torque & $1 \mathrm{kgf}-\mathrm{cm}$ \\
Hold torque & $1 \mathrm{kgf}-\mathrm{cm}$ \\
Weight & $20 \mathrm{~g}$ \\
\hline
\end{tabular}

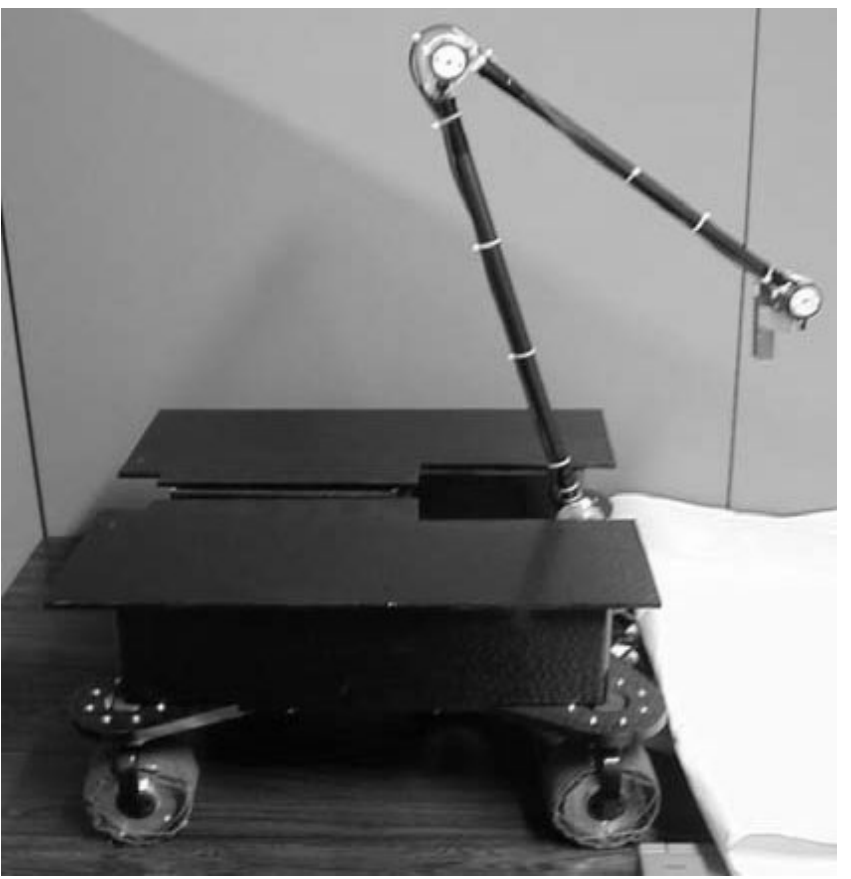

Figure 9. Manipulator mounted on Micro5.

problem. This system is realized to be simple and light in weight, because the design is based upon a simple four-wheel drive system.

\subsection{Experimental study}

To investigate the performance of the designed rover, the test-bed rover Micro5 was developed. Micro5 navigation strategy is based on both
Table 3. Specifications of smart manipulator.

\begin{tabular}{ll}
\hline Total length & $920 \mathrm{~mm}$ \\
Total weight & $1.45 \mathrm{~kg}$ \\
Conveyable weight & $300 \mathrm{~g}$ (on the Earth) \\
Actuator & Ultra-Sonic Motor \\
Reducer & Harmonic gear \\
\hline
\end{tabular}

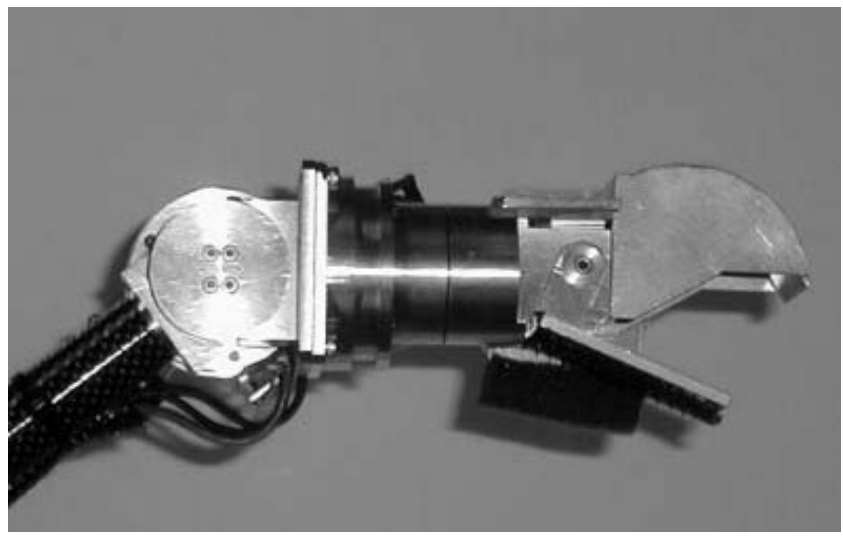

Figure 10. End-effector.

teleoperation and autonomous behavior. The performance on the mobility of Micro5 is demonstrated by teleoperation. Micro5 does not have any active steering mechanism. The surface of the Moon is covered with regolith so the steering is controlled by differential of left and right wheels. To turn easily, special tyres with spiral fin have been developed. Figure 6 shows the image sequences of some outdoor experiments. Micro5 can get over small craters and obstructions. The experimental results show that performance of the designed micro rover is satisfactory.

\section{Micro manipulator}

To perform in situ analysis or direct observation on lunar surface, manipulation is required. In this 

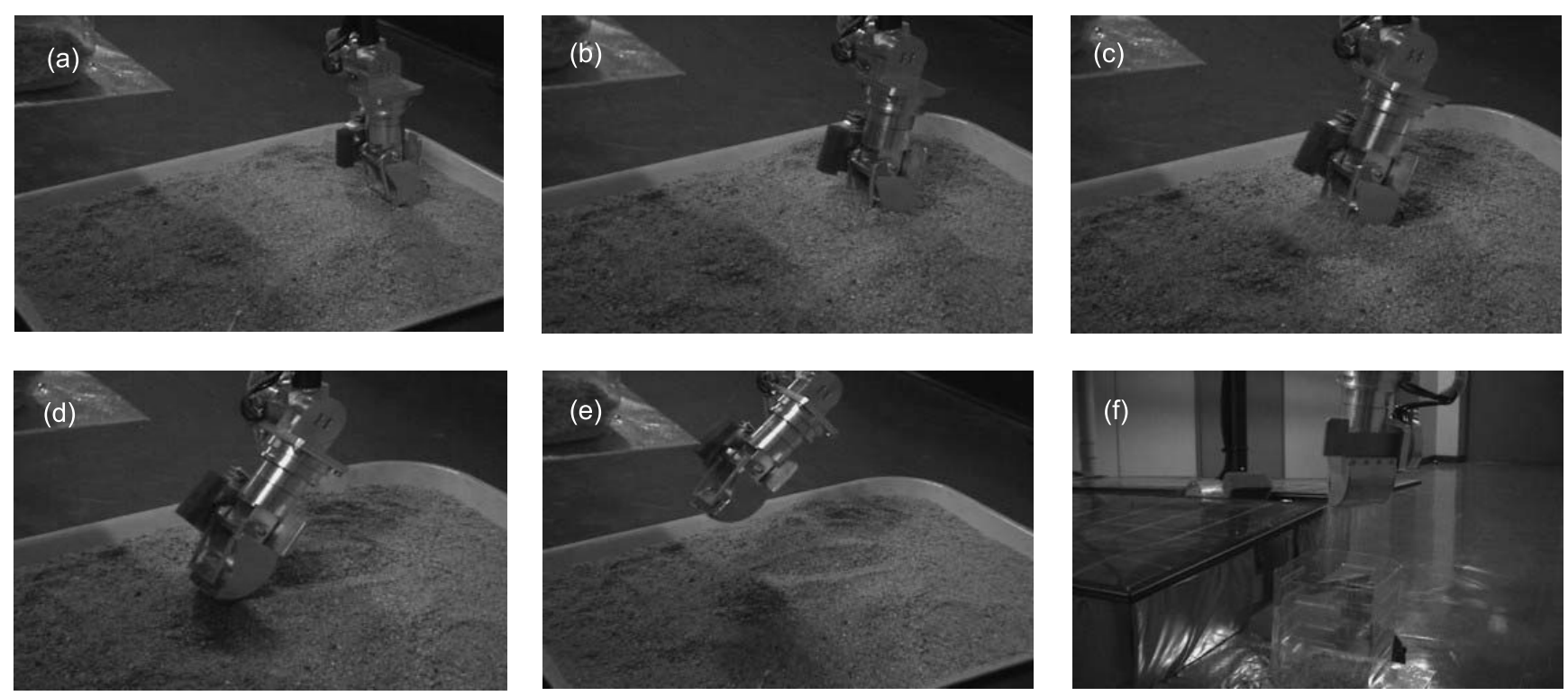

Figure 11. Experimental results on sample collection.
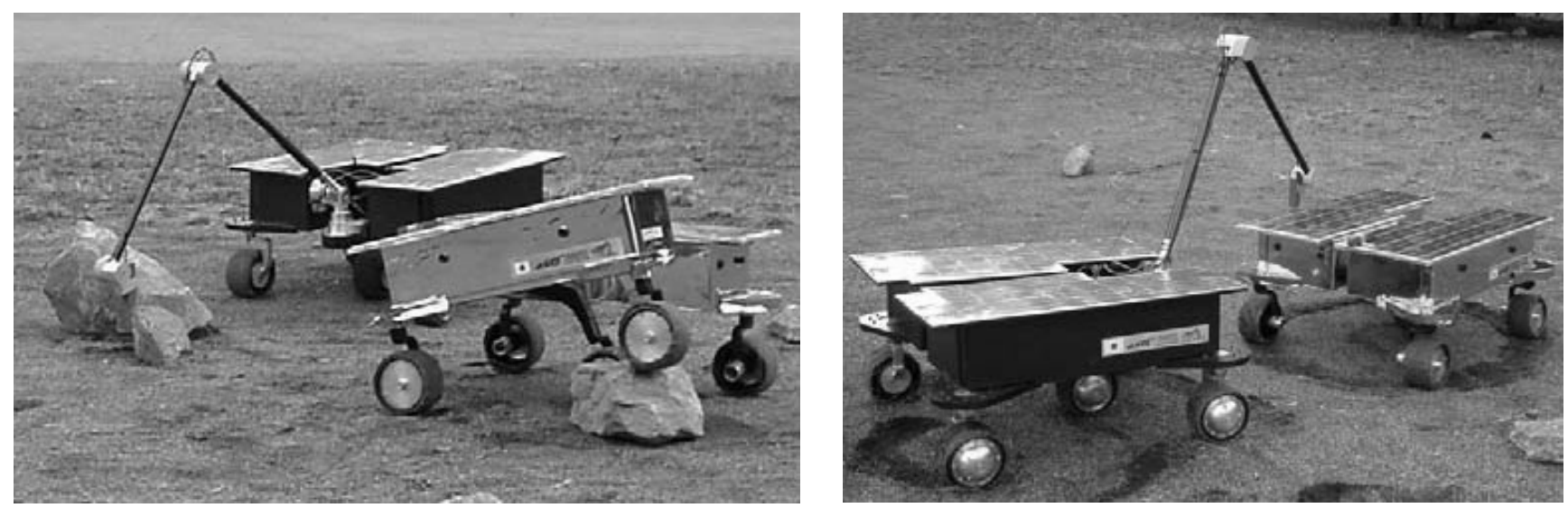

Figure 12. Co-operative exploration by micro rovers.

context, the science requirements and the design of lunar manipulator are discussed here.

\subsection{Requirements for manipulator}

Requirements for a manipulator in the rover mission are as follows:

- Observation of remote places (by a camera, etc.).

- Observation of environment surrounding a rover (including itself).

- Collecting samples for scientific observations.

- Approaching and contacting soils and rocks with scientific instruments such as spectrometers and imaging systems.

- Digging the ground or breaking samples into pieces.

- Removal of dust from the surface of samples.

- Functions as maintenance tools such as the cleaning tool for the solar panels, etc.
- 3D space measurement sensor, etc.

On the other hand, to use manipulators in space environment, various kinds of conditions listed below should be considered.

- Down sizing and weight reduction.

- Low electric power consumption.

- Long links for large work space and observation of remote places.

- Enough degree of freedom for missions.

- Installation of sensors such as cameras.

- Installation of end-effectors.

\subsection{Design of lunar manipulator}

Considering the abovementioned demands and requirements, we propose a smart manipulator for lunar rover. The structure of the designed manipulator is shown in figure 7 . The proposed 
manipulator has five degrees of freedom (5DOFs), because of some operations, for example, picking up samples and inserting a science equipment into topsoil.

\subsection{Ultra-sonic motor}

One of the solutions to reduce the power consumption might be a clutch put on a joint. However the clutch mechanism increases the weight of each joint and complexity of joint structures. High gear ratio is also one solution, but it is difficult to guarantee reasonable joint angles without a control system. We, therefore, believe that the best solution of this problem is to apply Ultra-Sonic Motor (USM) to the smart manipulator (Kunii 2001). The actuation principle of USM is an ultra-sonic wave, which makes a traveling wave on the surface of a stator. This traveling wave makes an elliptical orbital motion on a contact surface of a rotor, and the rotor is carried in the direction of the traveling wave. To make a torque on a rotor, it should have a large friction on an interface between a stator and a rotor. It means that USM can have a large static friction during an idle state, so that the USM works like a clutch. Furthermore, USM does not need any electrical and CPU power to stop rotation of a joint. USM and its specifications are shown in figure 8 and table 2 respectively. The specification shows that USM is the best solution on the point of low electrical power consumption and its weight.

\subsection{Development of micro manipulator}

We have developed a micro-manipulator with USM as shown in figure 9. The developed manipulator is mounted on the middle of the rover. The manipulator is located in the space between right and left body, where it does not disturb power generation of solar panels. The salient features of the manipulator are described in table 3. USM drives each joint with Harmonic Drive gear, and almost all of the links are made of carbon fiber plastics (CFRP). For sample collection, a new end-effector was developed as shown in figure 10. This endeffector has two kinds of functions, gripping and scooping. The experimental results for sample collection show the effectiveness of the end-effector as shown in figure 11.

The manipulator is automatically operated between the closed mode and the opened mode. When the manipulator is in the closed mode, the direction of a hand-eye camera is in front of Micro5. Therefore it is possible to obtain the front view image from Micro5. On the other hand, the workspace is mainly at the back of Micro5, when it is in the opened mode. It can also touch the solar panel side and it is possible to clean up dust on the panels. Figure 12 shows that two types of Micro5 are working on a collaborated mission together. An operator controls a manipulator on Micro5-02, which is shown on the left side of figure 12 , getting information from Micro5-01. Micro5-02 is inspecting solar panels on Micro5-01 with its hand-eye camera and an operator can get detailed images of solar panels by this collaboration as shown on the right side of figure 12 .

\section{Conclusions}

This paper presents the scheme of the Japanese lunar robotic exploration. A co-operative lander and rover based exploration is proposed. A lunar rover has also been studied for the future lunar missions requiring long traverses and rover-based science experiments. We have discussed a new design concept on the small lightweight rover with a novel mobility and smart manipulator. The lightweight manipulator operates with ultrasonic motors. Some experiments and demonstrations have shown that the performance of the lunar rover is satisfactory.

\section{Acknowledgements}

We thank Dr. Manabu Kato and Dr. Kohtaro Matsumoto for supporting this study on lunar robotics exploration.

\section{References}

Hapke B, Cassidy W and Wells E 1975 Effects of vaporphase deposition process on the optical, chemical and magnetic properties of the lunar regolith; Moon $\mathbf{1 3}$ 339-353.

Kubota T, Kuroda Y, Kunii Y 2001 Newly Developed Micro-rover System for Lunar Exploration; 10th Int. Conf. on Advanced Robotics, 451-456.

Kunii Y, Tada K, Kuroda Y, Kubota T and Nakatani T 2001 Micro-Manipulator actuated by ultra-sonic wave for planetary rover: Micro5, Sixth International Symposium on Artificial Intelligence, Robotics and Automation in Space, ASO20.

Nakatani I, Matsumoto K, Izumi T 2003 SELENE-B: Proposed Lunar Mission with Lander and Rover; 7th Int. Symposium on Artificial Intelligence and Robotics and Automation in Space, AS-11.

Nishiguchi K 2002 Obstacle Detection and Avoidance Method to Achieve a Soft Landing on the Moon and Planets; Trans. on the Society of Instruments and Control Engineers 38(4) 1-9.

Nozette S, Lichtenberg C L, Spudis P, Bonner R, Ort W, Malaret E, Robinson M and Shoemaker E M 1996 The Clementine Bistatic Radar Experiment; Science $\mathbf{2 7 4}$ 1495-1498.

Sasaki S, Kubota T, Okada T et al 2004a Scientific Exploration of Lunar Surface using a Rover in Japanese Future Lunar Mission; 35th Int. Conf. COSPAR, No.BO.2-0017. 
Sasaki S, Kubota T et al 2004b Rover-Lander Exploration on the Lunar Surface by SELENE-B: a Crater's Central Peak Window to the Lunar Interior; Int. Conf. on ISTS, No. 2004-k-3.

Tompkins S and Pieters C M 1999 Mineralogy of the lunar crust: results from Clementine; Meteoritics Planet. Sci. $3425-41$.
Weisbin C R, Lavery D, Rodriguez G 1997 Robotics Technology for Planetary Missions into the 21st Century; 5th Int. Symposium on Artificial Intelligence and Robotics and Automation in Space.

Wieczorek M A and Phillips R J 2000 The "Procellarum KREEP Terrane": Implications for mare volcanism and lunar evolution; J. Geophys. Res. 105 20,417-20,430. 